\title{
Existing and Emerging Technologies for Measuring Stable Isotope Labelled Retinol in Biological Samples: Isotope Dilution Analysis of Body Retinol Stores
}

Tom Preston

Stable Isotope Biochemistry Laboratory, Scottish Universities Environmental Research Centre, University of Glasgow, United Kingdom

\begin{abstract}
This paper discusses some of the recent improvements in instrumentation used for stable isotope tracer measurements in the context of measuring retinol stores, in vivo. Tracer costs, together with concerns that larger tracer doses may perturb the parameter under study, demand that ever more sensitive mass spectrometric techniques are developed. GCMS is the most widely used technique. It has high sensitivity in terms of sample amount and uses high resolution GC, yet its ability to detect low isotope ratios is limited by background noise. LCMSMS may become more accessible for tracer studies. Its ability to measure low level stable isotope tracers may prove superior to GCMS, but it is isotope ratio MS (IRMS) that has been designed specifically for low level stable isotope analysis through accurate analysis of tracer:tracee ratios (the tracee being the unlabelled species). Compound-specific isotope analysis, where GC is interfaced to IRMS, is gaining popularity. Here, individual ${ }^{13} \mathrm{C}$-labelled compounds are separated by GC, combusted to $\mathrm{CO}_{2}$ and transferred on-line for ratiometric analysis by IRMS at the ppm level. However, commercially-available ${ }^{13} \mathrm{C}$-labelled retinol tracers are $2-4$ times more expensive than deuterated tracers. For ${ }^{2} \mathrm{H}$-labelled compounds, GC-pyrolysis-IRMS has now become more generally available as an operating mode on the same IRMS instrument. Here, individual compounds are separated by GC and pyrolysed to $\mathrm{H}_{2}$ at high temperature for analysis by IRMS. It is predicted that GC-pyrolysis-IRMS will facilitate low level tracer procedures to measure body retinol stores, as has been accomplished in the case of fatty acids and amino acids. Sample size requirements for GC-P-IRMS may exceed those of GCMS, but this paper discusses sample preparation procedures and predicts improvements, particularly in the efficiency of sample introduction.
\end{abstract}

Key words: Retinol stores; Retinol isotopologues; GCMS; LCMSMS; GC-pyrolysis-IRMS 


\section{Introduction}

The application of non-hazardous stable isotope dilution techniques is recognised to be one of the most versatile means to quantify retinol stores in vivo, through a large range in retinol status from inadequate to subtoxic, and suitable for subjects of all ages [1]. This paper discusses some of the recent improvements in instrumentation necessary to undertake stable isotope tracer measurements in the laboratory, referring to recent developments in the broader fields of human protein and lipid metabolism that also apply stable isotope tracer methodology.

Stable isotope techniques provide a powerful means of assessing Vitamin A status in body stores and to follow their changes after nutritional intervention. The body has a relatively large intracellular retinol pool (up to one mmole), predominantly intrahepatic, with a slow turnover, yet the accessible pool (blood) is of low concentration (nmole/mL to sub-nmole $/ \mathrm{mL}$ ). This makes tracer quantitation technically demanding. Pioneering studies using radioactive ${ }^{14} \mathrm{C}$-labelled $\beta$-carotene in combination with high sensitivity accelerator MS [2], applied sub- $\mu$ mole tracer doses. As many national and international bodies no longer promote radio-isotope use in vivo for other than therapeutic reasons, the use of radioisotopes for such studies will remain of limited general utility. In contrast, early studies using stable isotope tracers applied doses of tens of micromoles that are considerably greater than the usual daily intake and likely to influence the parameter under study (retinol RDA in adult males is $900 \mu \mathrm{g}$ or $\sim 3 \mu$ moles; Haskell and co-workers [3] estimated a basal daily intake of $100 \mu \mathrm{g}$ retinol activity equivalent or $\sim 0.3 \mu$ moles in a study of Bangladeshi men). True tracer studies seek to make minimal changes in substrate concentration. In practice, for a high enrichment in the tracer dose (>90 moles \% excess $(\mathrm{MPE})$; where, $\mathrm{MPE}=100 \mathrm{x}$ moles tracer $/$ (moles tracer + moles tracee)), this criterion is often judged by observing tracer enrichment in the accessible free pool (in this case, plasma retinol) and ensuring its enrichment remains below 5 MPE.

When applying isotope dilution, precision and accuracy of analysis of the isotope ratio at isotopic equilibrium are key criteria. This may be a considerably lower enrichment than the maximum observed after giving a tracer dose. In the case of isotope dilution analysis of retinol stores, blood sample collection in adult subjects can last up to 21 days following tracer dosage. Longer sample collection periods of up to 48 days have been used in carotenoid bioavailability studies $[4,5,6]$. Although the enrichment of ${ }^{2} \mathrm{H}_{4}$ - retinol may reach a maximum of $1-2$ mole \% excess (MPE) in the circulation 1-2 days after an oral dose of $5 \mu$ mole retinyl acetate (RA), it may reduce to only 0.15-0.5 MPE after 3 days and 0.02-0.05 MPE after 21 days. A dose of $5 \mu$ mole $(1.6 \mathrm{mg}) \mathrm{RA}$ is already greater than the RDA in an adult male. A tenfold reduction in tracer dosage appears appropriate, more in subjects with marginal Vitamin A intake. Analytical methods have to perform well over two orders of magnitude in enrichment. In contrast, should interpretation of the enrichment after 3 days prove successful [7] instead of its analysis after 21 day [3], it has already been observed that the tracer dose and cost could be reduced to $20-50 \%$ of that used in a 21 day adult protocol [8]. Unless they impact measured tracer abundance, discussion of the details of the design and calculations underlying the different protocols in use to measure body retinol stores is outside the remit of this manuscript, which deals with quantifying tracer abundance For instance, models make assumptions which include the proportion of total body stores within the liver and the proportion of the tracer dose given that is absorbed. Also differing pathophysiology, such as inflammatory status, may reduce tracer absorption. It is thus recommended that inflammatory status be recorded when undertaking measurements of retinol status by stable isotope dilution methodology [9].

\section{Mass Spectrometry (MS) of Retinol Isotopologues}

\section{Gas Chromatography MS (GCMS)}

Early studies of retinol stores were accomplished using deuterated retinol isotopologues with GCMS analysis of samples prepared from plasma. Pioneering work by Furr and co-workers [10] used a large dose of $45 \mathrm{mg}(135 \mu \mathrm{mole}){ }^{2} \mathrm{H}_{4}$-retinyl acetate (RA) followed by GCMS analysis of free retinol. Free retinol is 'active' and not an ideal analyte for GC separation. Free retinol also fragments heavily under electron impact (EI) ionisation and isotope analysis was performed on $\mathrm{M}-\mathrm{H}_{2} \mathrm{O}^{+}$fragment ions which are produced at variable yield. Further refinements in retinol analysis by GC have since been made, all selecting retinol esters for analysis. Silyl esters are well-suited to GCMS analysis as they can show increased volatility, they produce sharp, well resolved peaks and the silyl group protects volatile molecules from fragmenting heavily during ionisation. Handelman and co-workers [11] prepared 
Table I: Theoretical natural abundance ratios of fragment ions of analytes using EI GCMS [39]

\begin{tabular}{lllllll}
\hline Analyte & $\begin{array}{l}\text { Fragment } \\
\text { Mass }\end{array}$ & $\begin{array}{l}\text { Fragment } \\
\text { C atoms }\end{array}$ & $\begin{array}{l}\text { Fragment } \\
\text { Si atoms }\end{array}$ & M+1:M & M+2:M & $\mathrm{M}+3: \mathrm{M}$ \\
\hline Bis-tBDMS-leucine & 302 & 14 & 2 & 0.261 & 0.102 & 0.017 \\
Bis-tBDMS-phenylalanine & 234 & 14 & 1 & 0.209 & 0.053 & 0.006 \\
tBDMS-phenylethylamine & 178 & 10 & 1 & 0.164 & 0.045 & 0.004 \\
tBDMS-palmitate & 313 & 18 & 1 & 0.250 & 0.067 & 0.010 \\
tBDMS-retinol & 255 & 19 & 0 & 0.209 & 0.021 & 0.001 \\
tBDMS-retinol & 400 & 26 & 1 & 0.337 & 0.090 & 0.016 \\
\hline
\end{tabular}

tertiary-butyldimethylsilyl ( $t$ BDMS) esters for analysis using EI ionisation on the GCMS. A high dose of $20 \mathrm{mg}(60 \mu \mathrm{mole}){ }^{2} \mathrm{H}_{4}$-retinyl acetate (RA) was applied and samples analysed with a limit of detection (LOD; throughout this manuscript, MPE units of stable isotope enrichment are used to describe LOD) of $0.2 \mathrm{MPE}$, allowing $>100$ fold range of enrichments from the maximum of 37.5 MPE. In contrast, Tang and co-workers [12] prepared trimethylsilyl (TMS) esters and analysed stable isotope enrichment on the GCMS under negative chemical ionisation (NCI). They quoted a LOD of $0.01 \mathrm{MPE}$ using a reduced tracer dosage of $1.5 \mathrm{mg}(4.5 \mu \mathrm{mole}){ }^{2} \mathrm{H}_{8}$-retinol RA in a procedure where the tracer reached a maximum of 2.2 MPE. Maximum tracer:tracee ratio in the Handelman protocol was 0.6 , equivalent to $37.5 \mathrm{MPE}$, thus both methods required to perform over a 200 -fold enrichment range. Two other differences in the analytical approaches in these studies should be commented upon. The $t$ BDMS esters used by Handelman [11] have proven more stable, being more resistant to hydrolysis than TMS esters. Tang and co-workers [12] noted that the formation of $t$ BDMS esters require more aggressive conditions than TMS esters, which may be detrimental to yield. However, newer $t$ BDMS donors with catalysts and alternative solvents have proven that milder derivatisation conditions are possible for a number of analytes, offering potential for future improvements. 'Harder' EI ionisation as used by Handelman [11] produces more ions than the 'softer' NCI ionisation used by Tang [12]. In contrast, $\mathrm{NCI}$ is more selective than EI, producing simpler mass spectra with prominent high mass ions suited to stable isotope ratio analysis [13]. Edwards and co-workers [14] have shown that TMS-retinol is also suitable for GCMS analysis under EI ionisation, by monitoring the molecular ion at 358 Th. TBDMS esters do not show as complex fragmentation under EI as say, retinyl acetate. They produce prominent high mass ions suitable for stable isotope analysis (Table I). Both TMS and $t$ BDMS esters appear suitable for stable isotope analy- sis in retinol. The lower LOD given by Tang and coworkers [12] does not mean the $t$ BDMS derivative as used by Handelman and co-workers [11] cannot be applied at a greatly improved LOD. Extending the LOD simply may not have been the priority of their study as the desired isotope dilution range had been achieved. What is perhaps more important is the reduced cost and dose used in the protocol described by Tang and co-workers [12]. The most important feature of any method is the signal to noise level which ultimately governs precision. This criterion includes instrumental parameters that contribute to the background signal, such as low MS resolution and detector noise.

Modern quadrupole MS have perhaps the greatest dynamic range of any MS type, yet they provide the most precise and accurate ratio measurement when peak areas are of similar amplitude. At very low enrichment the base peak can be orders of magnitude more abundant than that of the heavy isotopologue. Typical derivatised analytes in biomedical stable isotope tracer studies have 10-30 carbon atoms, the natural abundance of ${ }^{2} \mathrm{H},{ }^{13} \mathrm{C},{ }^{15} \mathrm{~N},{ }^{17} \mathrm{O},{ }^{18} \mathrm{O},{ }^{29} \mathrm{Si}$ and ${ }^{30} \mathrm{Si}$ all contribute to the $\mathrm{M}+1$ and $\mathrm{M}+2$ peaks. Table I gives the theoretical natural abundance of $\mathrm{M}+1$ and $\mathrm{M}+2$ and $\mathrm{M}+3$ for the bis- $t \mathrm{BDMS}-\mathrm{leucine}$, bis- $t \mathrm{BDMS}$ phenylalanine, $t \mathrm{BDMS}$-phenylethylamine, $t \mathrm{BDMS}$ palmitate and $t$ BDMS-retinol fragments formed under EI ionisation, all have been applied in human studies in a variety of isotopologue forms.

\section{Fragment ion and ionisation mode}

The relatively high natural abundance at $\mathrm{M}+1$ and $\mathrm{M}+2$ largely control the LOD of enrichment measurement of single atom tracers. The higher natural abundance at $\mathrm{M}+1$ and $\mathrm{M}+2$ of the amino acids and fatty acids reflects the fact that in addition to ${ }^{13} \mathrm{C}$, these fragments contain silicon atoms from the derivative, with a $4.7 \%$ natural abundance at $\mathrm{M}+1$ and $3.1 \%$ at $\mathrm{M}+2$, while the prominent retinol fragment at $255 \mathrm{Th}$ 
has lost its silicon atom during ionisation. This table also illustrates why use of multiple heavy atom tracers, such as ${ }^{2} \mathrm{H}_{3}$-leucine, ${ }^{2} \mathrm{H}_{5}$-phenylalanine, ${ }^{2} \mathrm{H}_{4}$ - and ${ }^{2} \mathrm{H}_{8}$ retinol is popular. $\mathrm{M}+3$ and higher isotopologues have greatly reduced interference from natural abundance, allowing improved LOD using GCMS (or LCMSMS) when analysing these tracers. Especially in the case of studies of human protein synthesis using amino acid tracers, several groups have extended the effective range of tracer dilution by using $\mathrm{M}+1$ or even $\mathrm{M}+2$ $[15,16,17,18]$ as proxy for base peak, as the resultant peak ratio is measured more readily. This approach allows the base peak to be over-scaled to achieve improved signal to noise ratio on the tracer peak and the tracer:tracee ratio $[16 ; 17]$. The approach may be further enhanced by selecting analytes and derivatives with favourable fragmentation patterns [18]. In the context of retinol isotopologue analysis by GCMS, Tang and co-workers [12] have used the $\mathrm{M}+1$ isotopologue as proxy for base peak to extend the LOD to 0.01 MPE. However, they did not explore the use of $\mathrm{M}+2$ as proxy. Slater and co-workers [17] showed a precision of $0.0005 \mathrm{MPE}$ (1 SD) when using the $\mathrm{M}+5 / \mathrm{M}+2$ peak ratio of $t$ BDMS-phenylethylamine to measure ${ }^{2} \mathrm{H}_{5}$-phenylalanine. This suggests that a LOD of the order of 0.002 MPE may ultimately be achievable for retinol isotopologues. This projection assumes that sample size is sufficient for optimal signal to noise ratio. Tang and co-workers [12] applied NCI to measure TMS-retinol isotopologues used only $0.2 \mathrm{~mL}$ plasma, whereas Handelman and co-workers [11] used $1 \mathrm{~mL}$ plasma.

\section{Interference}

It is very important to ensure that the region of the chromatogram where retinol elutes is free from isobaric interference from other volatiles. For instance, $\mathrm{M}+2$ and $\mathrm{M}+4$ of $t \mathrm{BDMS}$-retinol appear at 257 and $259 \mathrm{Th}$, respectively, which may both be selected for analysis of ${ }^{2} \mathrm{H}_{4}$-retinol. Free fatty acids readily produce $t$ BDMS esters with prominent M-57 peaks [19]. TBDMS-lauric acid (dodecanoic acid, which comprises some $2 \%$ of human fatty acids) has a prominent peak at $257 \mathrm{Th}$ with isotope peaks at $258 \mathrm{Th}$ and $259 \mathrm{Th}$, which are isobaric with $t$ BDMS-retinol fragments. It is important to ensure that sample processing removes the bulk of esterified and non-esterified fatty acids and that the GC temperature programme can resolve such interference. A minor fragment at $263 \mathrm{Th}$ in the $t \mathrm{BDMS}$-retinol EI mass spectrum [11], possibly $\mathrm{M}-\mathrm{C}_{10} \mathrm{H}_{17}$ which includes the hydrocarbon ring, may interfere with ${ }^{2} \mathrm{H}_{8}$-retinol analysis. If a longitudinal study design calls for both ${ }^{2} \mathrm{H}_{8}$ - and ${ }^{2} \mathrm{H}_{4}$-retinol isotopologues to be used, the molecular ion fragment series at $400 \mathrm{Th}$ could be used (Table I). Alternatively ${ }^{2} \mathrm{H}_{6}$-retinol, could be selected with ${ }^{2} \mathrm{H}_{4}$-retinol should a second tracer be warranted. Finally, it is also important to establish that the tracer stock is chemically and isotopically pure, and does not include traces of minor isotopologues that may interfere with analysis.

\section{Data reduction}

Two approaches exist to GCMS data acquisition and the estimation of stable isotope enrichment. The empirical approach monitors and acquires the peak areas of the unlabelled peak and one for each labelled isotopomer. In theory, some information is lost as small but predictable quantities of the tracer appear at adjacent masses, but signal to noise is improved by dwelling on the most important peaks. When using an empirical approach it is very important that unlabelled and labelled gravimetric standards are included in each batch of unknowns. Overall accuracy is improved if these are derived from the tracer stock used for human studies. As the latter are likely to be in the form of retinyl esters, this process would involve making the gravimetric dilutions of labelled and unlabelled esters, saponification under gentle conditions, followed by derivatisation. The alternative approach to MS data acquisition is to deconvolute the full mass envelope, calculating abundance by isotopologue distribution analysis [20]. In practice, when applying pure highlyenriched tracers purchased from a specialist supplier or synthesised in the laboratory, the empirical approach is more commonly used. In contrast, when applying low enrichment tracer materials, such as randomly labelled tracers produced during biosynthesis, isotopologue distribution analysis may be used to deconvolute complex mass spectra [20].

\section{Improved GC modes}

The past decade has seen continuous technical improvements in GC instrumentation and accessories. Capilliary GC has inherently high resolution. Apolar GC column phases, such as dimethyl polysiloxane and $5 \%$ phenyl $/ 95 \%$ dimethyl polysiloxane (DB1 and DB5 equivalents), are frequently used to separate silyl derivatives. Conventionally $0.25 \mathrm{~mm}$ ID columns were used, but 'fast GC' columns of $0.18 \mathrm{~mm}$ ID or less have become increasingly popular. These offer faster 
analytical cycles but they also offer improved resolution, whichever is the most important criterion. Large volume injection techniques have the potential to improve signal to noise by concentrating dilute samples in the GC injector. The have become more popular, not only through the use of dedicated large volume injectors, but also by using versatile multimode injectors that in combination with auto-injectors and inert retention gaps, allow larger injection volumes in standard injectors [21]. Finally, microfluidic devices such as Deans' switches are entering the GC market and can be used in several modes. These inert flow switches have very little effect on peak resolution and can be used simply to switch solvents and reagents to waste to protect the MS from contamination, or they can be used in 2D GC mode with a short medium polarity GC column phase such as a $50 \%$ phenyl polysiloxane $/ 50 \%$ dimethyl polysiloxane (DB50 type) downstream, allowing further enhanced resolution and improved signal to noise in the key sample peaks, such as $t$ BDMS-retinol. There is little doubt that further progress will be made in the coming years in GC injection technique, which is the interface between sample preparation and instrumental analysis.

\section{Sample preparation: Extraction and purification of plasma lipids}

The first step in developing any sample preparation procedure is to decide what class of molecule are destined for analysis. In the case of retinol analysis, the first decision is whether the analysis is to be simply that of free retinol or whether esterified retinol and carotenoids should also be studied. This consideration is outside the scope of this document which deals with the measurement of body retinol stores but general principles will be discussed.

The first step in processing blood plasma samples is to extract lipids while discarding plasma proteins. This can be a selective process by choice of solvent, where retinol is extracted while rejecting less polar lipids. Deuker and colleagues [22] used acetonitrile to precipitate proteins, extracting retinol from plasma samples and leaving behind less polar lipid classes. Automated column and plate-format devices are now available to facilitate this. Their use is commonplace in metabolomics and proteomics laboratories. Alternatively, if it is deemed necessary to extract retinyl esters and carotenoids along with retinol, conventional protein precipitation with ethanol, followed by hexane extraction of lipids can be applied [10;11]. Solvent extracts of lipids can be vacuum concentrated in readiness for the next phase of analysis. Retinyl esters can be analysed directly by LCMSMS procedures (see below) or hydrolysed to retinol (by alkaline hydrolysis / saponification) under as mild conditions as possible, prior to derivatisation and GCMS analysis. As the retinol ester fraction is usually at lower concentration than free retinol, some have analysed free retinol and a second fraction comprised of free and saponified retinyl esters in order to avoid the poorer signal to noise when analysing a retinyl ester fraction alone [20]. In practice, extraction of free retinol has been the aim of those studying the body's retinol stores, whereas an analysis of retinoid and carotenoid kinetics has been the objective of bioavailability studies.

\section{HPLC and /or Solid Phase Extraction purification}

Crude lipid extracts contain many lipid classes. As retinol is a minor component, extracts have to be purified further prior to derivatisation and GCMS analysis of retinol isotopologues. Most laboratories have used HPLC methodology to accomplish this. Reversed phase columns (predominantly C18 phases) have been applied with solvents of varying polarity. Some laboratories have resorted to two stage cleanup procedures applying two different isocratic mobile phases [23]. Others have used a single HPLC separation with more elaborate gradient separations [20], often in combination with a second purification step using Solid Phase Extraction (SPE) columns. SPE columns are disposable. They contain bonded phases similar to HPLC columns but of coarser particle size. They are optimised for sample flow under gravity, vacuum or mild pressure conditions. Several manufacturers make vacuum manifolds that can accommodate 10 or 20 samples in parallel. 96-well plate formats are also available. With a large variety of phases and the ability to elute samples in solvent systems of varying polarity and $\mathrm{pH}, \mathrm{SPE}$ has become the system of choice in many GCMS laboratories from pharmacology, to toxicology, to environmental monitoring laboratories. The fact that a single aminopropyl phase SPE column has been shown capable of separating all major plasma lipid classes when a series of elution solvents of decreasing polarity are used, is testament to the potential of this procedure [24]. As samples are processed in parallel, the avoidance of cross contamination is a major feature which is a real asset for stable isotope tracer studies. An SPE laboratory can be set up at much lower cost than an HPLC laboratory, which means that it is feasible to consider this as a satellite laboratory which part-processes samples for a MS 
laboratory which may be located at a distance or even in another country. This approach also avoids the issue of transporting human blood plasma over distances or national boundaries, as solvent extracts or dried extracts can be transported readily in small vials. In any event, all procedures adopted should be thoroughly tested to ensure sample integrity. As early as 1993, Deuker and co-workers [22] demonstrated that retinol extracts could be prepared in suitable purity for GCMS analysis following selective solvent extraction of plasma with a reversed phase SPE column to purify lipids followed by an aminopropyl SPE column to purify retinol.

GCMS is an extremely powerful technique with which to undertake stable isotope analysis in individual molecules. In other applications, GCMS is commonly used to assess analyte concentration, yet this has not usually been undertaken in the case of plasma retinol analysis where tracer enrichment has been under investigation. This is likely because HPLC has been used during sample clean up, where retinol concentration has been measured. Should SPE be used in place of HPLC to clean up samples prior to GCMS analysis, it is entirely feasible to add an internal standard to accomplish retinol concentration analysis as well as isotope analysis by GCMS, taking precautions to protect the sample from oxidation. Should a known quantity of internal standard of similar chemical nature to retinol, such as a long chain fatty alcohol, be added to plasma samples as these were processed, then retinol concentration could be assessed by conventional internal standard ratio analysis.

\section{Alternative MS approaches}

\section{Tandem MS}

High resolution tandem GCMSMS (triple quadrupole MS) is available in some laboratories. These instruments are capable of accurate analysis in complex samples which is facilitated by the extra MS dimension. Tandem MS are used for analysis of complex matrices such as blood plasma. They are marketed for their ability for high sample throughput as the analyst no longer needs to spend as much effort on sample preparation. However for the most accurate isotope analysis it is preferable to present the MS ion source with a clean sample to reduce matrix complexity, even if in practice this means increasing the duration of the analytical cycle. Very powerful tandem LCMS instruments (triple quadrupole or LCMSMS) are becoming widespread in clinical laboratories and 'omics research laboratories. They are far more common than tandem GCMS. These are costly facilities. However costs are reducing and installation of LCMSMS instrumentation in human nutrition research laboratories is underway. LCMSMS has great potential in retinol tracer studies and in those of fat soluble vitamins in general. It is very sensitive and can accommodate simultaneous analysis of carotenoids, retinyl esters and free retinol $[25,26$, 27]. LCMSMS can analyse compounds without derivatisation and, in tandem mode where the fragments analysed are of lower mass, it can potentially achieve lower LOD than GCMS when analysing isotope ratios. However, until instruments become more generally accessible in order for dedicated analytical cycles to be developed for low enrichment stable isotope analysis, it is unlikely to realise its full potential in isotope dilution studies. Current LCMSMS instruments are 4-10 times the price of GCMS.

\section{Isotope Ratio MS (IRMS)}

IRMS is a specialised form of MS which has been optimised for analysis of stable isotope natural abundance variations. It is very well suited to low enrichment tracer analysis. Briefly, IRMS features include: low resolution, magnetic sector, multiple detector $\mathrm{MS}$ with an EI ion source for simple gases, such as $\mathrm{CO}_{2}$ and $\mathrm{H}_{2}$. A family of continuous flow IRMS instruments have been developed since the introduction of on-line sample preparation [28], where sample gases are produced and analysed on-line in a helium carrier. Capillary GC can now be interfaced to IRMS in so-called compoundspecific isotope analysis [29]. The first of these instruments that become commercially available in the 1980 s was termed GC-combustion-IRMS (GC-C-IRMS), used a capillary with oxidation catalyst to combust samples on-line between GC separation and IRMS analysis [30]. As all analytes are converted to simple gases prior to IRMS analysis, it is very important to avoid interference by ensuring baseline separation in the GC. GC-C-IRMS was quickly exploited for low enrichment studies of amino acids, free fatty acids and glucose metabolism in human subjects [31, 32]. GC-CIRMS has been applied to retinol studies using biosynthesised ${ }^{13} \mathrm{C}-\beta$-carotene [33], ${ }^{13} \mathrm{C}_{2}$ - and ${ }^{13} \mathrm{C}_{4}$-retinol [23]. The development of equivalent techniques for deuterium analysis has lagged behind because of the greater technical challenge imposed by interference from the helium carrier and the low natural abundance of deuterium. However it is the latter that makes deuterium an attractive tracer. Its natural abundance (156 ppm) is far less than that of ${ }^{13} \mathrm{C}(10,815 \mathrm{ppm})$. GC-pyrolysis- 
Table II: Estimated LOD (MPE) for isotope dilution analysis of retinol isotopologues using the different MS techniques (based on the author's unpublished data and reference 30)

\begin{tabular}{lcccc}
\hline MS Technique & ${ }^{2} \mathrm{H}_{4}$-retinol & ${ }^{2} \mathrm{H}_{8}$-retinol & ${ }^{13} \mathrm{C}_{2}$-retinol & ${ }^{13} \mathrm{C}_{10}$-retinol \\
\hline GCMS $^{1}$ & 0.01 & 0.01 & 0.10 & 0.01 \\
LCMSMS $^{2}$ & 0.002 & 0.002 & 0.02 & 0.002 \\
GC-C-IRMS $^{3}$ & - & - & $<0.0011$ & $<0.0002$ \\
GC-P-IRMS $^{3}$ & $<0.0008$ & $<0.0004$ & - & - \\
\hline
\end{tabular}

Notes: ${ }^{1}$ Analysed as TMS or TBDMS retinol. $\mathrm{M}+2$ has higher natural abundance, so the ${ }^{13} \mathrm{C}_{2}$ isotopologue has a poorer LOD. The molecular ion fragment series at 400 Th may provide superior LOD than 255 Th, should the $t$ BDMS ester of the ${ }^{2} \mathrm{H}_{8}$-retinol isotopologue be used. ${ }^{2}$ Analysed underivatised. Performance assumed to match the best projection for GCMS. ${ }^{3}$ Analysed as the acetate ester. GC-C-IRMS and GC-P-IRMS analysis can be accomplished on the same instrument. Note that in all cases, LOD will be degraded at reduced sample size.

IRMS (GC-P-IRMS) was introduced in 1997 [34] and was demonstrated for deuterium analysis in individual volatiles. The approach was to interface a $\sim 1400^{\circ} \mathrm{C}$ pyrolysis oven between the GC and IRMS to convert all analytes quantitatively to $\mathrm{H}_{2}$, which is interfaced to an IRMS designed for on-line $\mathrm{H}_{2}$ analysis. Currently there are 4 manufacturers of GC-P-IRMS instruments. All of these instruments can be used in GC-C-IRMS and GC-P-IRMS modes. Compound specific isotope analysis was to be further developed to accommodate an $\mathrm{LC}$ interface to allow wet oxidation of ${ }^{13} \mathrm{C}$ to $\mathrm{CO}_{2}$ (LC-IRMS). This was designed for polar analytes such as glucose, separated in aqueous mobiles phase [35]. It is thus unlikely to be applied to the analysis of fat soluble vitamins which require organic mobile phases for separation, as these would be oxidised and lead to excessive $\mathrm{CO}_{2}$ production in the interface. $\mathrm{GC}-\mathrm{C} / \mathrm{P}$ IRMS instruments are 3-5 times the price of GCMS instruments, but performance with regard to low level stable isotope tracer studies offers immediate savings in tracer dose and cost.

As stated above, GCMS has shown a LOD of some 0.01 MPE in multi-atom tracers such as ${ }^{2} \mathrm{H}_{4}$-retinol, which can perhaps be extended as multiple atom tracers suffer much less interference from natural isotope abundance. GCMS measures the ratios of molecular ions or fragments. It is equally sensitive to a nmole of ${ }^{2} \mathrm{H}_{4}$-retinol and a nmole of ${ }^{2} \mathrm{H}_{8}$-retinol. In contrast, IRMS measures isotope ratios in simple gases, such as $\mathrm{H}_{2}$. Following combustion (or pyrolysis), all the carbon (hydrogen) atoms of the volatile derivative are converted to $\mathrm{CO}_{2}\left(\mathrm{H}_{2}\right)$ prior to IRMS analysis. For IRMS it is an advantage to select a volatile ester that adds a minimal number of carbon and hydrogen atoms. Such volatile esters are more polar than silyl esters and polar GC column phases would be appropriate for their separation. For instance, retinyl acetate (RA) could be prepared as analyte (if RA were selected as an additional internal standard, retinyl propionate could be used). A polar GC column such as a bonded phase polyethylene glycol (DB-Wax type) could be used for its separation. A wider bore $0.32 \mathrm{~mm}$ ID column may be selected to permit greater sample loading.

RA has $22 \mathrm{C}$ atoms and $32 \mathrm{H}$ atoms. Using ${ }^{13} \mathrm{C}_{2}$-RA as tracer, $2 / 22$ of the resulting $\mathrm{C}$ atoms in $\mathrm{CO}_{2}$ will be labelled. Using ${ }^{2} \mathrm{H}_{4}-\mathrm{RA}$ as tracer, 4/32 $\mathrm{H}$ atoms in $\mathrm{H}_{2}$ will be labelled and 8/32 atoms in the case of ${ }^{2} \mathrm{H}_{8}-\mathrm{RA}$. Thus, whereas multiple atom tracers can be used to improve signal to noise in GCMS by virtue of reduced natural abundance background, in IRMS more heavy atoms increase the signal directly. IRMS has inherently better precision of isotope analysis than quadrupole MS, being designed for precision of better than $1 \mathrm{ppm}$ excess $(<0.0001 \mathrm{MPE}$ in a simple gas). The comparison with GCMS can be made (see Table II). LOD is calculated as 3 times the standard deviation of the basal abundance. In practice, the maximum tracer dilution should remain above the limit of quantitation, which has been taken as ten times the standard deviation of the basal abundance [30].

The GCMS LOD is $0.01 \mathrm{MPE}$ for ${ }^{2} \mathrm{H}_{4}$-retinol and the same in ${ }^{2} \mathrm{H}_{8}$-retinol. The projected LOD of GCpyrolysis-IRMS is $<0.0008 \mathrm{MPE}(<0.0001 \times 32 / 4)$ for ${ }^{2} \mathrm{H}_{4}$-retinol and $<0.0004 \mathrm{MPE}(<0.0001 \times 32 / 8)$ for ${ }^{2} \mathrm{H}_{8}$ retinol. The calculations with respect to ${ }^{13} \mathrm{C}_{2}$-retinol are rather similar $<0.0011 \mathrm{MPE}(<0.0001 \times 22 / 2)$, improving with higher ${ }^{13} \mathrm{C}$ substitution. These calculations assume optimal sample size. Smaller sample sizes will erode LOD in either technique, but IRMS appears to offer an order of magnitude improvement in tracer detection compared with the best practice in GCMS. Also, sensitivity in GC-C/P-IRMS will benefit from all the advances in injection techniques that are described above for GCMS. This being said, it would be wise to assume that plasma samples of up to $2 \mathrm{~mL}$ should be processed for GC-C/P-IRMS analysis. 


\section{Tracer supply}

Pioneering studies of retinol tracer studies have been undertaken in laboratories with the facilities to synthesise stable isotope tracers, either through chemical synthesis routes $[10 ; 12 ; 23]$ or through biosynthesis [33]. Biosynthesis, where plants are labelled by ${ }^{13} \mathrm{CO}_{2}$ or ${ }^{2} \mathrm{H}_{2} \mathrm{O}$ during photosynthesis, is expensive but offers great potential as definitive studies of availability of the food matrix can be undertaken for carotenoids [4, 33, 36, 37] and other vitamins [38]. As these studies are expensive they are likely to remain the province of bioavailability studies. Isotope dilution studies of retinol stores require enriched RA as tracer. Deuterated RA isotopologues $\left({ }^{2} \mathrm{H}_{4}-\mathrm{RA},{ }^{2} \mathrm{H}_{6}-\mathrm{RA} \&{ }^{2} \mathrm{H}_{8}-\mathrm{RA}\right)$ and ${ }^{13} \mathrm{C}$-labelled RA isotopologues $\left({ }^{13} \mathrm{C}_{4}\right.$-RA, ${ }^{13} \mathrm{C}_{8}$ - RA $\&{ }^{13} \mathrm{C}_{10}$-RA) are now available commercially, with ${ }^{13} \mathrm{C}$-RA being 2-4 times the cost of ${ }^{2} \mathrm{H}-\mathrm{RA}$. To exploit the cost advantage of deuterated tracers, it is important to emphasise that GC-pyrolysis-IRMS is now a viable commercial instrumental solution to low level compound-specific deuterium analysis.

\section{Human protocols and longitudinal studies}

One of the most powerful uses of the stable isotope dilution technique is to measure retinol status before, and after a period of nutritional supplementation. In conditions when retinol status is low, its assessment using a minimal dose of tracer is important. With improvements in GCMS and the use of IRMS, smaller and cheaper doses are now possible. The same is likely to be so for LCMSMS, but its performance in terms of retinol isotope dilution analysis has yet to be demonstrated. Tang and co-workers [12] applied 4.5 umole of ${ }^{2} \mathrm{H}$-retinol isotopologues and GCMS. Using the costs contemporary with the Vitamin A Tracer Task Force Report [8] as a basis, a single dose of ${ }^{2} \mathrm{H}_{4}$-retinol for a measurement in adults would cost $\$ 12$. In a two tracer study to judge the effectiveness of an intervention, this is often combined with an equivalent dose of ${ }^{2} \mathrm{H}_{8}$ retinol which may cost a further $\$ 20$ per dose in adults. Tracer costs may thus be over $\$ 30$ per subject in an intervention study applying two tracers. Sample preparation and MS analysis may add approximately $\$ 120$. The twin concerns of high tracer cost and quantity can be addressed by using less tracer, while applying more sensitive methods of detecting stable isotope tracers, but the costs of doing the study will be determined by sample preparation and analysis. If IRMS is to be applied, this procedure cannot tell ${ }^{2} \mathrm{H}_{4}$-retinol apart from ${ }^{2} \mathrm{H}_{8}$-retinol as both are analysed as $\mathrm{H}_{2}$, but it does allow use of ${ }^{2} \mathrm{H}$-retinol in one phase followed by ${ }^{13} \mathrm{C}$ retinol in a second phase. Tanuminardjo [23] currently applies $<1 \mu$ mole dose of ${ }^{13} \mathrm{C}$-retinol with GC-C-IRMS analysis. The latter dose now appears possible using ${ }^{2} \mathrm{H}_{4}$ - or ${ }^{2} \mathrm{H}_{8}$-retinol and GC-pyrolysis-IRMS, showing IRMS can accommodate tracer doses approaching those of ${ }^{14} \mathrm{C}$-tracers used with accelerator MS [2, 5]. A longitudinal intervention study could be assessed with ${ }^{2} \mathrm{H}$-isotopologues alone with the proviso that an additional basal plasma sample is taken prior to the second tracer study. Alternatively, a combination of ${ }^{2} \mathrm{H}-\mathrm{RA}$ and ${ }^{13} \mathrm{C}-\mathrm{RA}$ isotopologues can be used.

\section{Conclusions}

LCMSMS will become more available for human nutrition studies but its full potential as a tool for isotope analysis is only likely to be realised when sufficient instrument time is made available for dedicated analytical cycles to be developed and demonstrated for isotope dilution assays. GCMS is a robust method with excellent sensitivity in terms of substrate quantity but finite improvements are foreseen for measuring isotope ratios. Further improvements in interfacing samples with GCMS are possible, but exactly the same improvements are possible for GC-C/P-IRMS. The latter requires more sample than GCMS but is capable of analysing smaller tracer enrichments. Specialist instrument modifications that have been proven in other areas, such as electron multiplier detectors for minor isotopes, could further reduce sample size requirements on IRMS instruments, but at a cost premium.

\section{References}

1. Tamunihardjo S (2004) Assessing Vitamin A status: past, present and future, J. Nutr., 134: 290S-293S.

2. Dueker SR, Lin Y, Buchhotz BA, Schneider PD, Lame MW, Segall HJ, Vogel JS and AJ Clifford (2000) Long-term kinetic study of $\beta$-carotene, using accelerator mass spectrometry in an adult volunteer J. Lipid Research 41: 1790-1800.

3. Haskell MJ, Jamil KM, Hassan F, Peerson JM, Hossain MI, Fuchs GJ and KH Brown (2004) Daily consumption of Indian spinach (Basella alba) or sweet potatoes has a sportive effect on total-body vitamin A stress in Bangladeshi men, Am. J. Clin. Nutr., 80: 705-714. 
4. Kurilich AC, Britz SJ, Clevidence BA and JA Novotny (2003) Isotopic labelling and LC-APCIMS quantification for investigating absorption of carotenoids and phyloquinone from kale (Brassica oleracea), J. Agric. Food Chem., 51(17): 4877-4883.

5. Hickenbottom SJ, Lembke SL, Deuker SR, Lin Y, Follet JR, Carkeet C, Buchholz BA, Vogel JS \& AJ Clifford (2002) Dual isotope test for assessing $\beta$-carotene cleavage to vitamin A in humans Eur. J. Nutr., 41: 141-147.

6. Lin Y, Deuker SR, Burri BJ, Neidlinger TR \& AJ Clifford (2000) Variability of the conversion of $\beta$-carotene to Vitamin $\mathrm{A}$ in women measured by using a double-tracer study design, Am. J. Clin. Nutr., 71: $1545-1554$.

7. Green MH \& JB Green (1990) Experimental and kinetic methods for studying Vitamin A dynamics in vivo. Method Enzymol 190: 304-317.

8. Vitamin A Tracer Task Force (2004) Appropriate uses of vitamin A tracer (stable isotope) methodology. http://www-naweb.iaea.org/nahu/nahres/documents/ vitatracer.pdf

9. Haskell MJ, Ribaya-Mercado JD and the Vitamin A tracer task force (2005) Handbook on Vitamin A Tracer dilution methods to assess status and evaluate intervention programs, Harvest Plus Monograph \#5. http://www-naweb.iaea.org/nahu/nahres/documents/ handbook.pdf

10. Furr HC, Amedee-Manesme O, Clifford AJ, Bergen HR III, Joes AD, Anderson DP and JA Olson (1989) Vitamin A concentrations in liver determined by isotope dilution assay with tetradeuterated vitamin A and by biopsy in generally healthy adult humans. Am. J. Clin. Nutr. 49: 713-716.

11. Handelman GJ, Haskell MJ, Jones AD and AJ Clifford (1993) An improved protocol for determining ratios of retinol- $d 4$ to retinol isolated from human plasma, Anal. Chem. 65: 2024-2028.

12. Tang G, Qin J and GG Dolnikowski (1998) Deuterium enrichment of retinol in humans determined by gas chromatography electron capture negative chemical ionisation mass spectrometry, J. Nutr. Biochem. 9: $408-414$

13. Wolfe RR (1992) Radioactive and stable isotopes in biomedicine: principles and practice of kinetic analysis, pp 471, Wiley-Liss, New York.

14. Edwards AJ, You C-S, Swanson JE and RS Parker (2001) A novel extrinsic reference method for assessing vitamin A value of plant foods, Am. J. Clin. Nutr. 74: 348-355.
15. Patterson BW, Zhang X-J, Chen Y, Klein S and RR Wolfe (1997) Measurements of very low stable isotope enrichments by gas chromatography/mass spectrometry: application to measurement of muscle protein synthesis, Metabolism 46 (8): 943-948.

16. Calder AG, Anderson SE, Grant I, McNurlan MA and PJ Garlick (1992) The determination of low d $\mathrm{d}_{5}$ phenylalanine enrichment (0.002-0.09 atom percent excess), after conversion to phenylethylamine, in relation to protein turnover studies by gas chromatography/electron ionization mass spectrometry, Rapid Commun. Mass Spectrom., 6: $421-424$.

17. Slater C, Preston T, McMillan DC, Falconer S and $\mathrm{KCH}$ Fearon (1995) GC/MS analysis of $\left[{ }^{2} \mathrm{H}_{5}\right]$ phenylalanine at very low enrichment: measurement of protein synthesis in health and disease, J. Mass Spectrom. 30: 1325-1332.

18. Preston $\mathrm{T}$ and AC Small (2010) Improved measurement of protein synthesis in human subjects using ${ }^{2} \mathrm{H}$-phenylalanine isotopomers and gas chromatography/mass spectrometry, Rapid Commun. Mass Spectrom., 24: 549-553.

19. Slater C, Hardieck M, Preston T and LT Weaver (1998) Analysis of tert-butyldimethylsilyl $\left[1-{ }^{13} \mathrm{C}\right]$ palmitic acid in stool samples by gas chromatographymass spectrometry with electron impact ionisation: comparison with combustion isotope-ratio mass spectrometry, J Chromatography B, 716: 1-6.

20. Ghavami A, Coward WA and LJC Bluck (2012) The effect of food preparation on the bioavailability of carotenoids from carrots using intrinsic labelling, British Journal of Nutrition, 107: 1350-1366.

21. MacDonald AJ, Small AC, Greig CA, Husi H, Ross JA, Stephens NA, Fearon KCH and T Preston (2013) A novel oral tracer procedure for measurement of habitual myofibrillar protein synthesis, Rapid Commun. Mass Spectrom., 27: 1769-1777.

22. Dueker SR, Lunetts JM, Jones AD and AJ Clifford (1993) Solid-phase extraction protocol for isolating retinol- $d_{4}$ and retinol from plasma for parallel processing for epidemiological studies, Clin. Chem,. 39 (11): 2318-2322.

23. Tamunihardjo SA (2001) Vitamin A status assessment in rats with ${ }^{13} \mathrm{C}_{4}$-retinyl acetate and gas chromatography/combustion/isotope ratio mass spectrometry, J. Nutr. 130: 2844-2849.

24. Kaluzny MA, Duncan LA, Merritt MV and DE Epps (1985) Rapid separation of lipid classes in high yield and purity using bonded phases columns, J. Lipid Research, 26: 135-140. 
25. Fleshman MK, Riedl KM, Novotny JA, Schwartz SJ and EH Harrison (2012) An LC/MS method for $d 8$ $\beta$-carotene absorption and its conversion to vitamin A in humans, J. Lipid Research 53: 820-827.

26. Oxley A, Berry P, Taylor GA, Cowell J, Hall MJ, Hesketh J, Lietz G \& AV Boddy (2014) An LC/MS/MS method for stable isotope dilution studies of $\beta$-carotene bioavailability, bioconversion, and vitamin A status in humans, J. Lipid Res. 55(2): $319-328$

27. Van Breemen RB, Nikolic $\mathrm{D}, \mathrm{Xu} \mathrm{X}$, Xiong $\mathrm{Y}$, Van Lieshout M, West CE \& AB Schilling (1998) Development of a method for quantitation of retinol and retinyl palmitate in human serum using highperformance liquid chromatography-atmospheric pressure chemical ionization-mass spectrometry, Journal of Chromatography A, 794: 245-251.

28. Preston T and NJP Owens (1983) Interfacing an automated elemental analyser with an isotope ratio mass spectrometer: the potential for fully automated total nitrogen and nitrogen-15 analysis, Analyst 108: 971-977.

29. Preston T. (1992) The measurement of stable isotope natural abundance variations. Plant, Cell and Environment, 15: 1091-1097.

30. Preston T \& Slater C, (1994) Mass Spectrometric Analysis of Stable Isotope Labelled Amino Acid Tracers. Proceedings of the Nutrition Society, 53: $363-372$.

31. Meier-Augenstein W, Watt PW and CD Langhans (1996) Influence of gas chromatographic parameters on measurement of ${ }^{13} \mathrm{C} /{ }^{12} \mathrm{C}$ isotope ratios by gasliquid chromatography-combustion isotope ratio mass spectrometry, J. Chromatography A, 752: $233-241$.

32. Yarasheski KE, Smith K, Rennie MJ and DM Bier (1992) Measurement of muscle protein synthetic rate by capillary gas chromatography/combustion isotope ratio mass spectrometry, Biological Mass Spectrometry 21: $486-490$.
33. Parker, RS, Swanson JE, Marmor B, Goodman KJ, Spielmand AB, Brenna JT, Viereck SM and WK Cranfield (1993) Study of $\beta$-carotene metabolism in humans using ${ }^{13} \mathrm{C}-\beta$-carotene and high precision isotope ratio mass spectrometry Ann. New York Acad. Sci. 691: 86-95.

34. Begley IA and CM Scrimgeour (1997) High-precision $\mathrm{d}^{2} \mathrm{H}$ and $\mathrm{d}^{18} \mathrm{O}$ measurement for water and volatile organic compounds by continuous-flow pyrolysis isotope ratio mass spectrometry, Anal. Chem., 69: $1530-1535$.

35. Krummen M, Hilkert AW, Juchelka D, Duhr A, Schluter H-J and R Pesch (2004) A new concept for isotope ratio monitoring liquid chromatography / mass spectrometry, Rapid Commun. Mass Spectrom. 18: $2260-2266$.

36. Grusak MA (1997) Intrinsic stable isotope labelling of plants for nutritional investigations in humans, J. Nutr. Biochem. 8: 164-171.

37. Tang G, Qin J, Dolnikowsji GG, Russell RM and MA Grusak (2005) Spinach or carrots can supply significant amounts of vitamin $\mathrm{A}$ as assessed by feeding with intrinsically deuterated vegetables Am. J. Clin. Nutr. 82: 821-828.

38. Bluck LJC (2009) Recent progress in stable isotope methods for assessing vitamin metabolism. Current Opinion in Clinical Nutrition and Metabolic Care, 12 (5): $495-500$.

39. http://yanjunhua.tripod.com/pattern1.htm

Professor Tom Preston

Stable Isotope Biochemistry Laboratory

Scottish Universities Environmental Research Laboratory

Rankine Avenue

East Kilbride

Glasgow, G75 0QF

United Kingdom

tom.preston@glasgow.ac.uk 